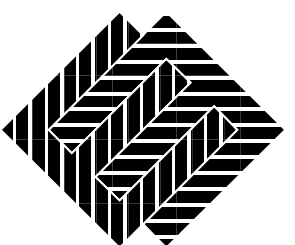

ENGINEERING MECHANICS

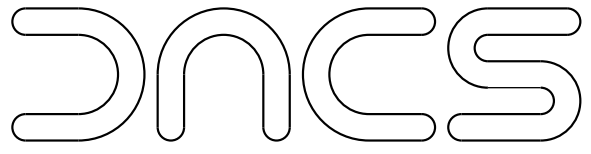

Delft Aerospace Computational Science

Added Mass Effects of Compressible and Incompressible Flows in Fluid-Structure Interaction

\author{
E.H. van Brummelen \\ e.h. vanbrummelen@tudelft.nl
}

Report DACS-09-001

January 2009 
Copyright (C) 2009: The copyright of this manuscript resides with the author.

All rights reserved. This work may not be translated or copied

in whole or in part without the written permission of the author.

TUD/LR/EM

Kluyverweg 1, 2629HS Delft, The Netherlands

P.O. Box 5058, 2600 GB Delft, The Netherlands

Phone: +31 152785460 Fax: +31 152611465

ISSN 1574-6992 


\title{
Added Mass Effects of Compressible and Incompressible Flows in Fluid-Structure Interaction
}

\author{
E.H. van Brummelen* \\ Delft University of Technology, Faculty of Aerospace Engineering
}

(Dated: March 12, 2008)

\begin{abstract}
The subiteration method which forms the basic iterative procedure for solving fluid-structureinteraction problems is based on a partitioning of the fluid-structure system into a fluidic part and a structural part. In fluid-structure interaction, on short time scales the fluid appears as an added mass to the structural operator, and the stability and convergence properties of the subiteration process depend significantly on the ratio of this apparent added mass to the actual structural mass. In the present paper, we establish that the added-mass effects corresponding to compressible and incompressible flows are fundamentally different. For a model problem, we show that on increasingly small time intervals, the added mass of a compressible flow is proportional to the length of the time interval, whereas the added mass of an incompressible flow approaches a constant. We then consider the implications of this difference in proportionality for the stability and convergence properties of the subiteration process, and for the stability and accuracy of loosely-coupled staggered timeintegration methods.
\end{abstract}

PACS numbers: 47.11.-j

Keywords: Fluid-structure interaction, added-mass effect, compressible and incompressible flow, subiteration

\section{INTRODUCTION}

The numerical simulation of the interaction of a flexible structure with a contiguous fluid flow is of critical importance to a multitude of applications, including the analysis of aero-elastic instabilities such as flutter in aerospace engineering $[1,2]$ and the investigation of cardiovascular disorders such as vulnerable plaques and aneurysms in biomechanics $[3,4]$. The basic iterative method for solving fluid-structure-interaction problems is subiteration. In the subiteration method, the fluid and solid subproblems are solved alternatingly, subject to complementary partitions of the interface conditions. In strongly-coupled partitioned schemes, the subiteration process is repeated until convergence to a prescribed tolerance. Alternatively, the subiteration method can be used as a preconditioner, for instance to a Krylov-subspace method [5, 6], or as a smoother in multigrid [7]. In loosely-coupled (or staggered) time-integration schemes, the subiteration procedure is performed only once per time step $[2,8,9]$.

On short time scales, the effect of the fluid on the structure can be represented as an added mass. The ratio of this apparent added mass to the structural mass is critical to the convergence and stability properties of the subiteration process. If the characteristic mass ratio exceeds one, then the subiteration process is unstable; see, e.g., [10]. The added-mass effect of incompressible flows has recently been studied in [10-12]. Heuristic methods to account for the added-mass effect in fluid-structureinteraction computations with very light structures such as large cargo parachutes have been proposed in $[13,14]$. However, improved understanding of these effects in en-

*Electronic address: e.h.vanbrummelen@tudelft.nl gineering computations would be beneficial. The addedmass effect of compressible flows is not well known. Moreover, despite the fact that there is a general concensus that the behaviour of subiteration is distinctly different for compressible and incompressible flows, it appears that the precise distinction is not well understood. This incomplete understanding has been the source of many miscommunications with regard to the stability properties of subiteration, and with regard to the accuracy and stability of staggered time-integration schemes, which depend strongly on the stability characteristics of the underlying subiteration procedure.

In the present paper, we investigate the difference between the added-mass effects pertaining to compressible and incompressible flows, and we consider the implications for the stability and convergence of the subiteration process, and for the stability and accuracy of staggered time-integration methods. Based on a model problem, viz., a fluid flow on a semi-infinite domain over a flexible panel in $2 \mathrm{D}$, we show that the added mass of a compressible flow is proportional to the length of the time step in the time-integration process, whereas the added mass of an incompressible flow approaches a constant as the time step vanishes. Consequently, regardless of the density of the fluid and the mass of the structure, the subiteration process is stable and convergent for compressible flows for sufficiently small time steps. For incompressible flows, this is not the case, and the subiteration method can remain unstable in the limit of vanishing time-step size. The distinct difference in the added-mass effect of compressible and incompressible flows, and in the corresponding properties of the subiteration method, is caused by the fact that for compressible flows the displacement of the interface affects the fluid only in the immediate vicinity of the interface, on account of the finite speed of sound in compressible fluids, whereas for 
incompressible fluids the displacement of the interface induces a global perturbation in the fluid. This qualitative difference between compressible and incompressible fluids applies identically to other fluid-structure-interaction problems. It is therefore anticipated that the results of this paper generalize mutatis mutandis to other, more complicated fluid-structure-interaction problems.

For incompressible flows, the model problem that we consider is a generalization of that in [10], in that we include convective and viscous effects. Our analysis conveys, however, that these effects are subordinate in the short time-scale limit and, hence, in this limit we retrieve the results of [10] for incompressible flow. The approach in this paper is based on formal Fourier analyses of linearized model problems, without regard for convergence of the Fourier series in the appropriate norms. The results can be provided with a rigorous footing, but this is beyond the scope of the present paper.

The contents of this paper are organized as follows: Section II presents the problem statement. In sections III and IV we derive the relation between the structural displacement and the corresponding pressure exerted by the fluid on the structure for the compressible-flow model and the incompressible-flow model, respectively. Section V investigates the stability and convergence properties of subiteration for the two flow types. In section VI we consider the implications of the distinct properties of subiteration for compressible and incompressible flows for the stability and accuracy of staggered time-integration methods. Section VII contains concluding remarks.

\section{PROBLEM STATEMENT}

To formulate the model problems, let $x, y$ and $t$ designate a horizontal spatial coordinate, a vertical spatial coordinate and a temporal coordinate, respectively. We consider an open space-time domain,

$Q_{\alpha}=\{(x, y, t): 0<t<T, 0<x<L, \alpha(x, t)<y<\infty\} ;$

see the illustration in Figure 1. The bottom boundary of $Q_{\alpha}$, which represents the interface between the compressible or incompressible fluid flow in $Q_{\alpha}$ and the structure, is given by

$$
\Gamma_{\alpha}=\{(x, y, t): 0<t<T, 0<x<L, y=\alpha(x, t)\} .
$$

The fluid models are elaborated in sections III and IV.

The structural model that we consider pertains to the flexural vibration of a beam:

$$
m \frac{\partial^{2} z}{\partial t^{2}}+\sigma^{2} \frac{\partial^{4} z}{\partial x^{4}}=p_{0}-\pi(x, t)
$$

with $m$ the mass of the beam per unit length, $z$ the vertical displacement, $\sigma$ the flexural rigidity, $p_{0}$ a prescribed exterior pressure, and $\pi$ the force exerted by the fluid on the structure.

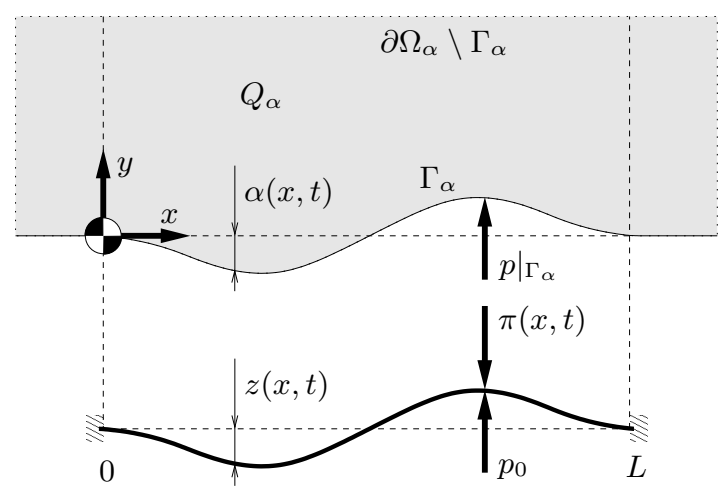

FIG. 1: Illustration of the panel problem: Temporal cross section with expanded interface region.

Denoting by $\left.p\right|_{\Gamma_{\alpha}}$ the pressure in the fluid at the interface, the fluid and the structure are connected by the dynamic and kinematic interface conditions,

$$
\pi(x, t)=\left.p\right|_{\Gamma_{\alpha}}, \quad \alpha(x, t)=z(x, t) .
$$

The fluid-flow models associate a unique pressure field $\pi$ with each admissible interface displacement field $\alpha$. We refer to the map $P: \alpha \mapsto \pi$ as the displacement-to-pressure ( $d t p$ ) operator corresponding to a particular flow model. For the panel-model problem that we consider, the customary subiteration approach for solving fluid-structure-interaction problems can be condensed into the following iterative procedure: Given an initial approximation of the structural displacement, $z_{0}$, repeat for $n=1,2, \ldots$,

$$
m \frac{\partial^{2} z_{n}}{\partial t^{2}}+\sigma^{2} \frac{\partial^{4} z_{n}}{\partial x^{4}}=p_{0}-P\left(z_{n-1}\right),
$$

To elucidate the problem considered in this paper, let us consider the particular case that (1) is provided with the homogeneous initial conditions

$$
z(x, 0)=0, \quad \partial_{t} z(x, 0)=0,
$$

and, moreover, suppose that the flow problem is furnished with initial and boundary conditions such that it admits a uniform flow with pressure $p_{0}$. The obvious solution to $(1)$ is then $z(x, t)=\bar{z}(x, t)=0$, and the corresponding solution of the flow problem is the uniform flow specified by the initial conditions. By adding a suitable partition of zero to (3), we obtain

$$
m \frac{\partial^{2}\left(z_{n}-\bar{z}\right)}{\partial t^{2}}+\sigma^{2} \frac{\partial^{4}\left(z_{n}-\bar{z}\right)}{\partial x^{4}}=-\left(P\left(z_{n-1}\right)-P(\bar{z})\right),
$$

If we restrict our considerations to displacements that are small in the appropriate norm, the right member of (5) can be linearized, and we obtain the following recursion relation for the iteration error $\varepsilon_{n}=z_{n}-\bar{z}$ in the subiteration process:

$$
m \frac{\partial^{2} \varepsilon_{n}}{\partial t^{2}}+\sigma^{2} \frac{\partial^{4} \varepsilon_{n}}{\partial x^{4}}=-P^{\prime} \varepsilon_{n-1}
$$


where $P^{\prime}$ designates the linearized dtp operator. Moreover, under the stipulation that the iterates $z_{n}$ comply with the initial conditions, it follows that the iteration errors $\varepsilon_{n}$ satisfies homogeneous initial conditions:

$$
\varepsilon_{n}(x, 0)=0, \quad \partial_{t} \varepsilon_{n}(x, 0)=0 .
$$

In the sequel of this paper, we derive the linearized dtp operators for a compressible flow and an incompressible flow model, and we examine the corresponding behaviour of the subiteration error in compliance with (6) and (7).

\section{COMPRESSIBLE FLOW MODEL}

We consider a compressible flow governed by the Euler equations,

$$
\frac{\partial \mathbf{q}}{\partial t}+\frac{\partial \mathbf{f}(\mathbf{q})}{\partial x}+\frac{\partial \mathbf{g}(\mathbf{q})}{\partial y}=0, \quad(x, y, t) \in Q_{\alpha},
$$

with

$$
\begin{aligned}
\mathbf{q} & :=\left(q_{1}, q_{2}, q_{3}, q_{4}\right), \\
\mathbf{f}(\mathbf{q}) & :=\left(q_{2}, \frac{q_{2}^{2}}{q_{1}}+p(\mathbf{q}), \frac{q_{2} q_{3}}{q_{1}}, \frac{q_{2}\left(p(\mathbf{q})+q_{4}\right)}{q_{1}}\right), \\
\mathbf{g}(\mathbf{q}) & :=\left(q_{3}, \frac{q_{3}^{2}}{q_{1}}+p(\mathbf{q}), \frac{q_{2} q_{3}}{q_{1}}, \frac{q_{3}\left(p(\mathbf{q})+q_{4}\right)}{q_{1}}\right) .
\end{aligned}
$$

In $(8 \mathrm{~b}), q_{1}, q_{2}, q_{3}$ and $q_{4}$ denote the density, horizontal momentum, vertical momentum and total energy of the fluid, respectively. The system (8) is closed by the equation of state

$$
p(\mathbf{q}):=(\gamma-1)\left(q_{4}-\frac{1}{2}\left(q_{2}^{2}+q_{3}^{2}\right) / q_{1}\right),
$$

with $\gamma=1.4$.

At the interface, the fluid flow complies with the flow tangency condition

$$
\frac{\partial \alpha}{\partial t}+\frac{q_{2}}{q_{1}} \frac{\partial \alpha}{\partial x}-\frac{q_{3}}{q_{1}}=0 .
$$

The boundary conditions on the complement $\partial \Omega_{\alpha} \backslash \Gamma_{\alpha}$ will not be further elaborated.

To derive the linearized dtp operator corresponding to $(8)$, we consider small deflections:

$$
\alpha_{\epsilon}=0+\epsilon \alpha^{\prime}, \quad \epsilon \rightarrow 0 .
$$

Accordingly, we assume that the fluid solution can be formally expanded as $\mathbf{q}_{\epsilon}=\mathbf{q}_{0}+\epsilon \mathbf{q}^{\prime}+O\left(\epsilon^{2}\right)$, where the generating solution $\mathbf{q}_{0}$ corresponds to a uniform horizontal flow with density $\rho_{0}>0$, horizontal velocity $U_{0} \geq 0$ and pressure $p_{0}$,

$$
\mathbf{q}_{0}(x, y, t)=\left(\rho_{0}, \rho_{0} U_{0}, 0, \frac{1}{2} \rho_{0} U_{0}^{2}+p_{0} /(\gamma-1)\right) .
$$

One easily verified that (11) indeed satisfies (8) and (9) for $\alpha=0$. In addition, we assume that $\mathbf{q}_{\epsilon}$ is isentropic and irrotational. The first-order perturbation in the fluid solution can then be written as

$$
\mathbf{q}^{\prime}=\left(\rho^{\prime}, \rho^{\prime} U_{0}+\rho_{0} \partial_{x} \varphi^{\prime}, \rho_{0} \partial_{y} \varphi^{\prime}, E^{\prime}\right),
$$

where the potential $\varphi^{\prime}$ complies with the linearized fullpotential equation

$$
U_{0}^{2} \frac{\partial^{2} \varphi^{\prime}}{\partial x^{2}}+2 U_{0} \frac{\partial^{2} \varphi^{\prime}}{\partial x \partial t}+\frac{\partial^{2} \varphi^{\prime}}{\partial t^{2}}-C_{0}^{2}\left(\frac{\partial^{2} \varphi^{\prime}}{\partial x^{2}}+\frac{\partial^{2} \varphi^{\prime}}{\partial y^{2}}\right)=0
$$

with $C_{0}:=\sqrt{\gamma p_{0} / \rho_{0}}$ the speed of sound corresponding to the reference state. The energy perturbation $E^{\prime}$ is irrelevant in the sequel. The density perturbation $\rho^{\prime}$ it related to the potential by

$$
\rho^{\prime}=-\left(\rho_{0} / C_{0}^{2}\right)\left(\partial_{t} \varphi^{\prime}+U_{0} \partial_{x} \varphi^{\prime}\right) .
$$

Moreover, upon expanding the pressure according to $p\left(\mathbf{q}_{\epsilon}\right)=p\left(\mathbf{q}_{0}\right)+\epsilon p^{\prime}+O\left(\epsilon^{2}\right)$, it holds that $p^{\prime}=C_{0}^{2} \rho^{\prime}$. The flow-tangency condition (9) yields the first-order condition

$$
\frac{\partial \alpha^{\prime}}{\partial t}+U_{0} \frac{\partial \alpha^{\prime}}{\partial x}-\frac{\partial \varphi^{\prime}}{\partial y}=0
$$

It is to be noted that (12) and (14) hold on the unperturbed domain $Q_{0}$ and on the unperturbed interface $\Gamma_{0}$, respectively.

The Green's function for the wave equation (see, for instance, Refs. [15, p.473], [16, p.520]) enables us to express the pressure perturbation at $\Gamma_{0}$ in accordance with (12) and (14) as $\left.p^{\prime}\right|_{\Gamma_{0}}=P^{\prime} \alpha^{\prime}$, with the operator $P^{\prime}$ according to

$$
P^{\prime}=\pi^{-1} \rho_{0} C_{0} \Psi \Xi \Psi
$$

where

$$
\begin{aligned}
& (\Xi \psi)(x, t)=\int_{0}^{t} \int_{\mathbb{R}} \psi(\xi, \tau) \\
& \times \frac{H\left(C_{0}(t-\tau)-\left|(x-\xi)-U_{0}(t-\tau)\right|\right)}{\sqrt{C_{0}^{2}(t-\tau)^{2}-\left|(x-\xi)-U_{0}(t-\tau)\right|^{2}}} d \xi d \tau,
\end{aligned}
$$

with $H(\cdot)$ the Heaviside function and

$$
\Psi \varepsilon= \begin{cases}\partial_{t} \varepsilon+U_{0} \partial_{x} \varepsilon & \text { if }(x, t) \in] 0, L[\times] 0, T[ \\ 0 & \text { otherwise }\end{cases}
$$

It is noteworthy that the Heaviside function restricts the domain of integration to the triangle

$$
\left\{\tau<t, x-\left(U_{0}+C_{0}\right)(t-\tau)<\xi<x-\left(U_{0}-C_{0}\right)(t-\tau)\right\},
$$

which constitutes the projection of the domain of dependence associated with (12) for the space/time coordinate $(x, t)$ onto $\Gamma_{0}$. Equation (15) represents the linearized dtp operator corresponding to the considered compressible flow model. 
To facilitate the interpretation of the added-mass effect associated to (15), we derive the Fourier symbol of the operator (15). To this end, we first derive the Fourier symbol of the integral operator $\Xi$. Let us consider an isolated Fourier mode

$$
\psi(x, t)=\hat{\psi}(\kappa, \omega) \exp (i \kappa x+i \omega t) .
$$

Upon inserting (17) in (15b), and restricting the domain of integration in accordance with (16), we obtain

$$
(\Xi \psi)(x, t)=\hat{\Xi}(\kappa, \omega, x, t) \hat{\psi}(\kappa, \omega) \exp (i \kappa x+i \omega t),
$$

where the Fourier symbol $\hat{\Xi}$ is given by

$$
\begin{aligned}
& \hat{\Xi}(\kappa, \omega, x, t)=\int_{0}^{t} \int_{x-\left(U_{0}+C_{0}\right)(t-\tau)}^{x-\left(U_{0}-C_{0}\right)(t-\tau)} e^{-i(\kappa(x-\xi)+\omega(t-\tau))} \\
& \quad \times\left(C_{0}^{2}(t-\tau)^{2}-\left|(x-\xi)-U_{0}(t-\tau)\right|^{2}\right)^{-1 / 2} d \xi d \tau
\end{aligned}
$$

We introduce the transformations

$$
\begin{aligned}
& (\theta, \eta) \mapsto(\xi, \tau)=\left(x-\left(U_{0}-C_{0} \sin \theta\right) \eta, t-\eta\right), \\
& (r, \zeta) \mapsto(\kappa, \omega)=r 2 t^{-1}\left(C_{0}^{-1} \cos \zeta, \sin \zeta\right) .
\end{aligned}
$$

Note that the factor $C_{0}^{-1}$ is prerequisite in (20b) to ensure dimensional consistency. By means of (20) and the partition of unity $1=\sin ^{2} \theta+\cos ^{2} \theta$, the integral (19) can be condensed into

$$
\hat{\Xi}=t \int_{-\pi / 2}^{\pi / 2} \frac{\sin \beta}{\beta} \exp (-i \beta) d \theta,
$$

where

$$
\beta(r, \zeta, \theta)=r(M \cos \zeta+\sin \zeta-\cos \zeta \sin \theta),
$$

with $M=U_{0} / C_{0}$ the Mach number. Noting that $\left|\beta^{-1} \sin \beta\right| \leq 1$ for all $\beta \in \mathbb{R}$, it follows from the CauchySchwartz inequality that the Fourier symbol of $\Xi$ can be bounded as

$$
\begin{aligned}
|\hat{\Xi}(\kappa, \omega, x, t)| & \leq t\left\|(\cdot)^{-1} \sin (\cdot)\right\|_{L^{2}(-\pi / 2, \pi / 2)} \\
& \times\|\exp (-i(\cdot))\|_{L^{2}(-\pi / 2, \pi / 2)} \leq \pi t .
\end{aligned}
$$

For the operator $\Psi$ according to (15c) we simply obtain:

$$
(\Psi \psi)(x, t)=\hat{\Psi}(\kappa, \omega) \hat{\psi}(\kappa, \omega) \exp (i \kappa x+i \omega t)
$$

with $\hat{\Psi}=i\left(\omega+U_{0} \kappa\right)$. The Fourier symbol of the composite operator (15a) is the product of the Fourier symbols of the operators in the composition. Hence, we obtain the following upper bound for the Fourier symbol of the linearized dtp operator (15) associated with the compressible flow problem:

$$
|\hat{P}| \leq \rho_{0} C_{0} t\left|\omega+U_{0} \kappa\right|^{2} .
$$

In particular, in the analysis of the added-mass effect, we shall be interested in short time intervals or, equivalently, high frequencies. In this context, it is to be noted that (23) yields $|\hat{P}| \lesssim \rho_{0} C_{0} t \omega^{2}$ in the high-frequency limit $\omega \rightarrow \infty$. The Fourier symbol of this high-frequency limit can be associated with an added mass

$$
\mu_{\mathrm{c}}=\rho_{0} C_{0} t
$$

Hence, the added mass corresponding to the compressible flow is time dependent and, specifically, the added mass $\mu_{\mathrm{c}}$ is proportional to $t$.

Let us allude to the fact that the added mass $\mu_{\mathrm{c}}$ in (24) admits an intuitive physical interpretation: because pressure perturbations travel at the speed of sound $C_{0}$, the displacement of the interface has a local effect on the fluid, and only affects the fluid in a region within distance $C_{0} t$ of the interface. The mass corresponding to this region (per unit length) is precisely $\mu_{\mathrm{c}}$.

\section{INCOMPRESSIBLE FLOW MODEL}

We consider an incompressible flow governed by the Navier-Stokes equations,

$$
\begin{aligned}
\partial_{t} u+\partial_{x} u u+\partial_{y} u v+\partial_{x} p-\nu \Delta u & =0, \\
\partial_{t} v+\partial_{x} u v+\partial_{y} v v+\partial_{y} p-\nu \Delta v & =0, \\
\partial_{x} u+\partial_{y} v & =0
\end{aligned}
$$

where $u$ and $v$ represent the horizontal and vertical velocity components, respectively, $p$ denotes the pressure divided by the (homogeneous) fluid density $\rho_{0}, \nu$ is the dynamic viscosity and $\Delta$ designates the Laplace operator.

At the interface, the flow is assumed to obey slip boundary conditions. This implies that the flow complies with the tangency condition

$$
\frac{\partial \alpha}{\partial t}+u \frac{\partial \alpha}{\partial x}-v=0
$$

and, moreover, that the tangential component of the normal traction vanishes,

$$
\mathbf{n}_{\alpha} \cdot \nabla \mathbf{u} \cdot \mathbf{t}_{\alpha}+\mathbf{t}_{\alpha} \cdot \nabla \mathbf{u} \cdot \mathbf{n}_{\alpha}=0
$$

where $\mathbf{n}_{\alpha}$ and $\mathbf{t}_{\alpha}$ denote the unit normal vector and the unit tangential vector to $\Gamma_{\alpha}$, respectively, $\boldsymbol{\nabla}=\left(\partial_{x}, \partial_{y}\right)$ and $\mathbf{u}=(u, v)$. The boundary conditions on $\partial \Omega_{\alpha} \backslash \Gamma_{\alpha}$ will be elaborated in passing.

We are concerned with small deflections $\alpha_{\epsilon}$ conforming to (10) and, accordingly, we assume that the flow solution can be formally expanded as

$$
(u, v, p)_{\epsilon}=(u, v, p)_{0}+\epsilon(u, v, p)^{\prime}+O\left(\epsilon^{2}\right) .
$$

where the generating solution $(u, v, p)_{0}=\left(U_{0}, 0, p_{0}\right)$ again corresponds to a uniform horizontal flow. Upon 
inserting the expansion in (25a) and collecting terms of $O(\epsilon)$, we obtain the first-order conditions

$$
\begin{aligned}
\partial_{t} u^{\prime}+U_{0} \partial_{x} u^{\prime}+\partial_{x} p^{\prime}-\nu \Delta u^{\prime} & =0 \\
\partial_{t} v^{\prime}+U_{0} \partial_{x} v^{\prime}+\partial_{y} p^{\prime}-\nu \Delta v^{\prime} & =0 \\
\partial_{x} u^{\prime}+\partial_{y} v^{\prime} & =0 .
\end{aligned}
$$

These conditions hold on $Q_{0}$. The boundary conditions (26) and (27) moreover imply that $u^{\prime}$ and $v^{\prime}$ comply with the following first-order conditions on $\Gamma_{0}$ :

$$
\frac{\partial \alpha^{\prime}}{\partial t}+U_{0} \frac{\partial \alpha^{\prime}}{\partial x}-v^{\prime}=0
$$

and

$$
\frac{\partial u^{\prime}}{\partial y}+\frac{\partial v^{\prime}}{\partial x}=0
$$

For notational convenience, we introduce the condensed notation $\mathbf{q}^{\prime}(x, y, t)=\left(u^{\prime}, v^{\prime}, p^{\prime}\right)(x, y, t)$. Instead of deriving an explicit expression for the linearized dtp operator corresponding to (29)-(31), we establish its Fourier symbol. To this end, we regard an isolated Fourier component of the interface displacement,

$$
\alpha^{\prime}(x, t)=\hat{\alpha}(\kappa, \omega) \exp (i \kappa x+i \omega t)
$$

and a corresponding velocity/pressure perturbation

$$
\mathbf{q}^{\prime}(x, y, t)=\hat{\mathbf{q}}(k, \omega) \exp (i \kappa x+i \omega t+s y) .
$$

We stipulate that the velocity and pressure perturbations vanish as $y \rightarrow \infty$. This implies that the functions $s:=s(\kappa, \omega)$ must have strictly negative real part. Upon inserting (33) into (29), we obtain

$$
\hat{\mathbf{N}}(k, \omega) \cdot \hat{\mathbf{q}}(k, \omega) \exp (i \kappa x+i \omega t+s y)=0,
$$

where the Fourier symbol $\hat{\mathbf{N}}(k, \omega)$ of the system $(29)$ is defined by

$$
\hat{\mathbf{N}}(k, \omega)=\left(\begin{array}{ccc}
\hat{H}(k, \omega) & 0 & i \kappa \\
0 & \hat{H}(k, \omega) & s \\
i \kappa & s & 0
\end{array}\right)
$$

with $\hat{H}(k, \omega)=i \omega+i U_{0} \kappa+\nu\left(\kappa^{2}-s^{2}\right)$. Therefore, (33) complies with (29) if and only if

$$
\hat{\mathbf{q}}(k, \omega) \in \operatorname{kernel}(\hat{\mathbf{N}}(k, \omega)) .
$$

Equation (36) admits nontrivial solutions under the strict condition that

$$
\operatorname{det}(\hat{\mathbf{N}}(k, \omega))=\left(\kappa^{2}-s^{2}\right) \hat{H}(k, \omega)=0 .
$$

It then follows that (33) satisfies (29) provided that

$$
\hat{\mathbf{q}}(k, \omega) \in \operatorname{span}\left\{\left(i \kappa,-|\kappa|,-i\left(\omega+U_{0} \kappa\right)\right)\right\}, s=-|\kappa|,
$$

or

$$
\hat{\mathbf{q}}(k, \omega) \in \operatorname{span}\{(s,-i \kappa, 0)\}, \hat{H}(k, \omega)=0 .
$$

A solution to (29)-(31) with $\alpha^{\prime}$ specified by (32) can be obtained by combining the modes (38)-(39):

$$
\begin{aligned}
\mathbf{q}^{\prime} & =\hat{\alpha}\left[\frac{\left(\omega+U_{0} k\right)\left(\sigma^{2}+\kappa^{2}\right)}{|\kappa|\left(\kappa^{2}-\sigma^{2}\right)} \exp (-|\kappa| y)\left(\begin{array}{c}
-\kappa \\
-i|\kappa| \\
\left(\omega+U_{0} \kappa\right)
\end{array}\right)\right. \\
& \left.+\frac{2\left(\omega+U_{0} k\right) \kappa}{\kappa^{2}-\sigma^{2}} \exp (\sigma y)\left(\begin{array}{c}
-\sigma \\
i \kappa \\
0
\end{array}\right)\right] \exp (i \kappa x+i \omega t),
\end{aligned}
$$

with $\sigma(\kappa, \omega)= \pm \sqrt{\kappa^{2}+i\left(\omega+U_{0} \kappa\right) / \nu}$, subject to the restriction that the real part of $\sigma$ is negative. Recalling that the pressure divided by the density corresponds to the third component of (40), we obtain the following Fourier symbol for the linearized dtp operator corresponding to the incompressible flow:

$$
\hat{P}(\kappa, \omega)=\rho_{0}\left(-\frac{\left(\omega+U_{0} k\right)^{2}}{|\kappa|}+i \frac{2 \nu \kappa^{2}\left(\omega+U_{0} \kappa\right)}{|\kappa|}\right) .
$$

It is to be noted that the high-frequency limit of (41) yields $\hat{P} \sim-\rho_{0}|\kappa|^{-1} \omega^{2}$ as $\omega \rightarrow \infty$. This symbol can be associated with an added mass $\rho_{0}|\kappa|^{-1}$. In fact, the wave number can only assume values $\kappa=k \pi / L, k \in \mathbb{N}$, on account of the structural boundary conditions $\alpha(0, t)=$ $\alpha(L, t)=0$. Hence, the largest-wave-length component $(k=1)$ is dominant, and for this component it holds that $\hat{P} \sim-\mu_{\mathrm{i}} \omega^{2}$ as $\omega \rightarrow \infty$, where the added mass is defined by:

$$
\mu_{\mathrm{i}}=\rho_{0} L / \pi \text {. }
$$

Equation (42) conveys that the added mass corresponding to the incompressible flow is independent of time. It is noteworthy that the added mass (42) is consistent with that derived in [10], in the appropriate limit.

To provide a physical explanation for the difference in the added-mass effect for compressible and incompressible flows, we note that the mode (40) is global. Hence, whereas for compressible flows the effect of the displacement of the interface on the fluid is confined to a region within distance $C_{0} t$ of the interface (see section III), for incompressible flows the fluid is affected throughout its entire domain.

Let us moreover note that the convective part and the viscous part of $\hat{P}$ according to (41) are proportional to $\omega$, whereas the added-mass part is proportional to $\omega^{2}$. Hence, convective effects and viscous effects are subordinate to the added-mass effect in the limit $\omega \rightarrow \infty$.

\section{STABILITY AND CONVERGENCE OF SUBITERATION}

Equipped with the Fourier symbols of the linearized dtp operators, we can establish the behaviour of the iteration error according to (6) for the compressible and 
incompressible flows. Let us consider an isolated Fourier component of the iteration error:

$$
\varepsilon_{n}(x, t)=\hat{\varepsilon}_{n}(\kappa, \omega) \exp (i \kappa x+i \omega t),
$$

Upon inserting (43) in equation (6), we obtain the relation $\left|\hat{\varepsilon}_{n}(\kappa, \omega)\right| \leq \varrho(\kappa, \omega)\left|\hat{\varepsilon}_{n-1}(\kappa, \omega)\right|$, where the contraction number $\varrho$ is defined by:

$$
\varrho(\kappa, \omega)=\frac{|\hat{P}(\kappa, \omega)|}{\left|-m \omega^{2}+\sigma^{2} \kappa^{4}\right|} .
$$

Again restricting our consideration to high frequencies, it follows that the contraction number is bounded from above as $\varrho \leq \mu / m$ as $\omega \rightarrow \infty$, where $\mu$ refers to the added mass according to (24) and (42) for the compressible flow and incompressible flow, respectively, and equality holds in the incompressible case. Let us note that the following results extend without further modifications to other structural-stiffness operators, as for fixed $\kappa$ the contribution corresponding to the structural-stiffness operator to (44) vanishes in the limit $\omega \rightarrow \infty$. This argument has also been used in [12]. If $\varrho \leq 1$, the Fourier amplitudes $\hat{\varepsilon}_{n}$ form a non-increasing sequence and, hence, the subiteration process is stable. Moreover, if $\varrho<1$, the subiteration process is formally convergent, and $\varrho$ determines the rate of convergence. For the compressible and incompressible flows, equations (24), (42) and (44) lead to the following estimates for the corresponding contraction numbers:

$$
\begin{aligned}
& \varrho_{\mathrm{c}} \leq \frac{\rho_{0} C_{0} t}{m}+O\left(\omega^{-1}\right), \\
& \varrho_{\mathrm{i}}=\frac{\pi^{-1} \rho_{0} L}{m}+O\left(\omega^{-1}\right),
\end{aligned}
$$

as $\omega \rightarrow \infty$.

The estimates in (45) elucidate the fundamental difference in the properties of the subiteration method for compressible and incompressible flows. In computational methods, the subiteration procedure is generally applied to resolve the aggregated fluid-structure system within each time step of a time-integration process, i.e., the iteration (3) is repeated within each time step until the iteration error is inferior to a certain prescribed tolerance. Hence, within a time step, the sequence of iteration errors complies with (6) and (7), and we implicitly restrict our consideration of the iteration error to the time interval $0 \leq t \leq \delta t$, where $\delta t$ denotes the time step in the time-integration process. The upper bound (45a) then yields $\varrho_{\mathrm{c}} \leq \rho_{0} C_{0} \delta t / m$. In particular, this implies that for compressible flows the convergence behaviour of the subiteration process improves if the time step is reduced and, specifically, $\varrho_{\mathrm{c}} \rightarrow 0$ as $\delta t \rightarrow 0$. Let us remark that this behaviour has also been established for the piston problem in [17]. Consequently, for all settings of the structural mass $m$ and the fluid density $\rho_{0}$, there exists a strictly positive time step $\delta t^{*}$ such that the subiteration process is stable for all $\left.\delta t \in] 0, \delta t^{*}\right]$. Moreover, if the time-step size is reduced by a certain factor, then the convergence rate of the subiteration process improves by that same factor. For incompressible flows, this is not the case. For increasingly small time steps, i.e., in the limit $\delta t \rightarrow 0$, the contraction number converges towards the strictly positive, time-step-independent highfrequency limit in (45b). Therefore, if the characteristic fluid-structure mass ratio $\mu_{\mathrm{i}} / m$ exceeds one, the subiteration method is unstable, regardless of the time step. ${ }^{1}$

The above results have been established on the basis of the continuum problem. If a particular temporal discretization scheme is considered, then the structure of the estimates in (45) remains intact, although the precise values can be different. We refer to [12] for an overview of the effects of temporal discretization schemes on the stability of the subiteration procedure for fluid-structure interaction with incompressible flow.

\section{STAGGERED TIME-INTEGRATION METHODS}

The aforementioned fundamental difference in the convergence properties of the subiteration process for compressible and incompressible flows also carries important consequences for the suitability of staggered (also referred to as loosely-coupled or partitioned) time-integration procedures, i.e., time-integration methods in which the subiteration step is performed only once per time step; see, for instance, $[2,8,9]$. We regard a partition of the time interval under consideration, $0<t<T$, into time steps $t_{i-1}<t<t_{i}$ of uniform length $\delta t=t_{i}-t_{i-1}$ $(i=1,2, \ldots, T / \delta t)$. Within each time step, the aggregated fluid-structure system can be condensed into

$$
\mathcal{A} w_{i}=\mathcal{B} w_{i-1},
$$

with

$$
w_{i}=\left(\begin{array}{c}
q_{i} \\
z_{i}
\end{array}\right), \quad \mathcal{A}=\left(\begin{array}{ll}
A_{11} & A_{12} \\
A_{21} & A_{22}
\end{array}\right), \quad \mathcal{B}=\left(\begin{array}{cc}
B_{11} & 0 \\
0 & B_{22}
\end{array}\right),
$$

where $q_{i}$ and $z_{i}$ represent the variables pertaining to the discrete approximation of the fluid and structure solutions on interval $i$, and $A_{11}, A_{12}, A_{21}$ and $A_{22}$ denote the discretized fluid operator, kinematic condition, dynamic condition and structural operator, respectively. The operators $B_{11}$ and $B_{22}$ extract the initial conditions for the fluid and structure subsystems on interval $i$ from the approximation on the previous time interval. Of course, on the first interval the right member of (46) is replaced by a vector corresponding to the prescribed initial conditions.

\footnotetext{
1 In principle, this statement requires somewhat more care, because it is not a-priori obvious that (45b) does not represent an upper bound, attained in the limit $\omega \rightarrow \infty$. A more precise analysis of (44) with $\hat{P}$ according to (41) reveals that this is not the case.
} 
For simplicity, we assume that the operators $\mathcal{A}$ and $\mathcal{B}$ are linear, which is appropriate for the ensuing error analysis.

Let us assume that the system (46) has been solved inexactly on the previous time interval, $i-1$. In particular, the result on interval $i-1$ contains an error $\delta w_{i-1}$. This error propagates to an error $\delta w_{\mathrm{p}, i}$ on interval $i$ via the initial conditions. Hence, on account of the inexact solution on interval $i-1$, equation (46) is replaced by:

$$
\mathcal{A}\left(w_{i}+\delta w_{\mathrm{p}, i}\right)=\mathcal{B}\left(w_{i-1}+\delta w_{i-1}\right)
$$

By virtue of the assumed linearity of (47), the propagated error can be expressed in terms of the error on interval $i-1$ as $\delta w_{\mathrm{p}, i}=\mathcal{L} \delta w_{i-1}$ with $\mathcal{L}=\mathcal{A}^{-1} \mathcal{B}$. Note that the inverse operator $\mathcal{A}^{-1}$ is well defined under the standing assumption that the fluid-structure problem is well posed.

Application of the subiteration procedure to (47) leads to the following sequence of approximations: given an initial estimate $w_{i, 0}$, for $n=1,2, \ldots$,

$$
\begin{aligned}
\left(\begin{array}{cc}
A_{11} & 0 \\
A_{21} & A_{22}
\end{array}\right)\left(\begin{array}{l}
q_{i, n} \\
z_{i, n}
\end{array}\right)= & \left(\begin{array}{cc}
B_{11} & 0 \\
0 & B_{22}
\end{array}\right)\left(\begin{array}{l}
q_{i-1}+\delta q_{i-1} \\
z_{i-1}+\delta z_{i-1}
\end{array}\right) \\
& -\left(\begin{array}{cc}
0 & A_{12} \\
0 & 0
\end{array}\right)\left(\begin{array}{l}
q_{i, n-1} \\
z_{i, n-1}
\end{array}\right) .
\end{aligned}
$$

Note that the fluid and structure approximations with index $n$ in fact depend exclusively on the structural approximation with index $n-1$. Hence, to initialize the procedure, it is sufficient to prescribe $z_{i, 0}$. We define the local iteration error by $\delta w_{i, n}=w_{i, n}-\left(w_{i}+\delta w_{\mathrm{p}, i}\right)$. Upon adding a suitable partition of zero to (48), we obtain the error-amplification relation

$$
\left(\begin{array}{cc}
A_{11} & 0 \\
A_{21} & A_{22}
\end{array}\right)\left(\begin{array}{l}
\delta q_{i, n} \\
\delta z_{i, n}
\end{array}\right)=-\left(\begin{array}{cc}
0 & A_{12} \\
0 & 0
\end{array}\right)\left(\begin{array}{l}
\delta q_{i, n-1} \\
\delta z_{i, n-1}
\end{array}\right) .
$$

From (49), it follows that $\delta w_{i, n}=\mathcal{Q} \delta w_{i, n-1}$ with

$$
\mathcal{Q}=\left(\begin{array}{cc}
0 & -A_{11}^{-1} A_{12} \\
0 & A_{22}^{-1} A_{21} A_{11}^{-1} A_{12}
\end{array}\right)
$$

Hence, by recursion, $\delta w_{i, n}=\mathcal{Q}^{n} \delta w_{i, 0}$.

Suppose that the initial approximation on each time interval is obtained by means of prediction, i.e., by extrapolation of the approximation on the previous time interval. In particular,

$$
w_{i, 0}=\mathcal{E}\left(w_{i-1}+\delta w_{i-1}\right),
$$

where $\mathcal{E}$ represents the extension operator:

$$
\left(\mathcal{E} w_{i-1}\right)(x, t)=w_{i-1}(x, t+\delta t) \quad 0<t<\delta t .
$$

The extension (52) is well defined for finite-element approximations in time. For finite-difference approximations, it can be defined via interpolating polynomials. Assuming that in each time step the subiteration process is terminated after $\bar{n}$ iterations, the cumulative iteration error $\delta w_{i}$ in the final result on interval $i$ is composed of the propagated error and the local iteration error at iteration $\bar{n}$. From (47)-(51), we then obtain the sequence of identities:

$$
\begin{aligned}
\delta w_{i} & =\delta w_{i, \bar{n}}+\delta w_{\mathrm{p}, i}=\mathcal{Q}^{\bar{n}} \delta w_{i, 0}+\delta w_{\mathrm{p}, i} \\
& =\mathcal{Q}^{\bar{n}}\left(\mathcal{E}\left(w_{i-1}+\delta w_{i-1}\right)-\left(w_{i}+\delta w_{\mathrm{p}, i}\right)\right)+\delta w_{\mathrm{p}, i} \\
& =\mathcal{Q}^{\bar{n}}(\mathcal{E}-\mathcal{L}) w_{i-1}+\left(\mathcal{Q}^{\bar{n}}(\mathcal{E}-\mathcal{L})+\mathcal{L}\right) \delta w_{i-1} .
\end{aligned}
$$

The final identity in (53) is a consequence of $w_{i}=\mathcal{L} w_{i-1}$ and $\delta w_{\mathrm{p}, i}=\mathcal{L} \delta w_{i-1}$.

From (53) it follows by recursion that:

$$
\delta w_{i}=\sum_{k=1}^{i}\left(\mathcal{Q}^{\bar{n}}(\mathcal{E}-\mathcal{L})+\mathcal{L}\right)^{i-k} \mathcal{Q}^{\bar{n}}(\mathcal{E}-\mathcal{L}) w_{k-1}
$$

and, by the triangle inequality,

$$
\left\|\delta w_{i}\right\|=\sum_{k=1}^{i}\left\|\mathcal{Q}^{\bar{n}}(\mathcal{E}-\mathcal{L})+\mathcal{L}\right\|^{i-k}\|\mathcal{Q}\|^{\bar{n}}\|\mathcal{E}-\mathcal{L}\|\left\|w_{k-1}\right\|
$$

Recalling that $t_{i}=i \delta t$, we replace $i-k$ in the exponent in $(55)$ by $\left(t_{i}-t_{k}\right) / \delta t$. A necessary condition for boundedness of the right member of (55) in the limit $\delta t \rightarrow 0$ is:

$$
\left\|\mathcal{Q}^{\bar{n}}(\mathcal{E}-\mathcal{L})+\mathcal{L}\right\| \leq 1+\vartheta \delta t \quad \text { as } \quad \delta t \rightarrow 0
$$

for some positive constant $\vartheta$. The exponential term in (55) can then be bounded as

$$
\left\|\mathcal{Q}^{\bar{n}}(\mathcal{E}-\mathcal{L})+\mathcal{L}\right\|^{i-k} \leq(1+\vartheta \delta t)^{\left(t_{i}-t_{k}\right) / \delta t} \sim e^{\vartheta\left(t_{i}-t_{k}\right)}
$$

as $\delta t \rightarrow 0$. It is to be remarked that the provision $(56)$ does not hold for $\bar{n}=0$, because any appropriate norm of the extrapolation operator $\|\mathcal{E}\|$ exceeds 1 as $\delta t \rightarrow 0$. In particular, this implies that the analysis below does not hold if only extension is applied, or if the subiteration process is non-convergent or if convergence is too slow. In such circumstances, the right member of (55) becomes unbounded as $\delta t \rightarrow 0$.

Proceeding under the assumption (56), it follows from (55) and (57) that:

$$
\left\|\delta w_{i}\right\| \leq C \delta t^{-1} e^{\vartheta t_{i}}\|\mathcal{Q}\|^{\bar{n}}\|\mathcal{E}-\mathcal{L}\| \sup _{k}\left\|w_{k}\right\|,
$$

for some constant $C$ independent of $\delta t$, as $\delta t \rightarrow 0$. Suppose that the extension operator corresponds to an $m$-th order extrapolation. Then for sufficiently smooth functions $\|\mathcal{E}-\mathcal{L}\|=O\left(\delta t^{m}\right)$. Moreover, on account of the fact that $\left\|w_{k}\right\|$ pertains to a time interval of length $\delta t$, it holds that $\left\|w_{k}\right\|=O\left(\delta t^{1 / 2}\right)$. Therefore,

$$
\left\|\delta w_{i}\right\| \leq \bar{C}\left(t_{i}\right)\|\mathcal{Q}\|^{\bar{n}} O\left(\delta t^{m-1 / 2}\right),
$$

for some exponentially increasing function $\bar{C}(t)$, independent of $\delta t$.

The error $\delta w_{i}$, which is induced by the inexact solution of the aggregated fluid-structure system on the intervals 
with index $\leq i$, is to be compared to the discretization error on interval, i.e., the difference between the resolved (monolithic) discrete solution, and the actual continuum solution. Suppose that the monolithic discrete approximation corresponding to (46) yields an approximation to the solution of the fluid-structure system with formal temporal order of accuracy $m$, i.e., for sufficiently smooth solutions its holds that the approximation error on each time interval conforms to

$$
\left\|w_{i}-\bar{w}\right\| \leq C \delta t^{m}\|\bar{w}\|=O\left(\delta t^{m+1 / 2}\right),
$$

as $\delta t \rightarrow 0$, where $\bar{w}$ represents the continuum solution. The additional factor $1 / 2$ in estimate $(60)$ originates from the fact that the measure of the considered time interval is proportional to $\delta t$.

The upper bound (59) enables us to clarify the distinctly different properties of staggered time-integration procedures for compressible and incompressible flows. For compressible flows, $\|\mathcal{Q}\|$ is proportional to $\delta t$. In section $\mathrm{V}$ this proportionality has been established for the map $\delta z_{i, n-1} \mapsto \delta z_{i, n}$; cf. (45a). However, specifically, the norm of the map between the structure displacement and the fluid state, $\delta z_{i, n-1} \mapsto \delta q_{i, n}$, is proportional to $\delta t$, and the norm of the map between the fluid state and the structure displacement, $\delta q_{i, n} \mapsto \delta z_{i, n}$, is proportional to 1 as $\delta t \rightarrow 0$. Upon inserting the proportionality $\|\mathcal{Q}\| \propto \delta t$ in (59), it follows that for a compressible flow the iteration error on interval $i$, i.e., the error relative to the monolithic result, is bounded as:

$$
\left\|\delta w_{i}\right\| \leq C_{\mathrm{a}}\left(t_{i}\right) \delta t^{m+\bar{n}-1 / 2}
$$

as $\delta t \rightarrow 0$, for some exponentially increasing function $C_{\mathrm{a}}(t)$, independent of $\delta t$. For a staggered timeintegration method, $\bar{n}=1$ and, therefore, the cumulative iteration error is of the same order as the discretization error in the monolithic result; cf. (60). Hence, the staggered procedure possesses the same order of accuracy as the underlying monolithic method, but with a different constant of proportionality. As a digression, we note that for $\bar{n}=2$, the cumulative iteration error is one order higher than the discretization error. Consequently, in the limit $\delta t \rightarrow 0$, the result obtained with 2 subiterations per time step is identical to the monolithic results.

For incompressible flows, staggered time-integration methods behave distinctly different. In the incompressible case, the norm of $\|\mathcal{Q}\|$ converges to a positive constant in the limit $\delta t \rightarrow 0$. For $\bar{n}=1$, the global iteration error thus remains $O\left(\delta t^{m-1 / 2}\right)$ and, hence, the order of accuracy of a result obtained by a staggered method is one order lower than that of the underlying monolithic method. In fact, assuming that the subiteration process is convergent, the number of subiterations per time step must increase as $\bar{n} \propto|\log \delta t|$ as $\delta t \rightarrow 0$ to obtain a method which yields the same order of accuracy as a monolithic approach.

The distinct properties of $\|\mathcal{Q}\|$ for compressible and incompressible flows is also pertinent in relation to the condition (56). For compressible flows, $\|\mathcal{Q}\| \propto \delta t$ in the limit $\delta t \rightarrow 0$. Therefore, condition (56) is fulfilled for $\bar{n} \geq 1$ under the solitary provision that

$$
\|\mathcal{L}\|=1+O(\delta t) \quad \text { as } \quad \delta t \rightarrow 0,
$$

independent of the extrapolation operator. This implies that if (62) holds, then the solution of the staggered scheme cannot grow unbounded in finite time, on account of the upper bound (57). For incompressible flows, this is not the case, because $\|\mathcal{Q}\|$ does not vanish as $\delta t \rightarrow 0$.

\section{CONCLUSION}

To examine the difference between the added-mass effects of compressible and incompressible flows, we considered the model problem of flow in a semi-infinite domain over a flexible panel in 2D. We derived the displacementto-pressure operator which relates the pressure exerted by the fluid on the structure to the structural displacement for a compressible flow governed by the Euler equations and for an incompressible flow governed by the Navier-Stokes equations. For the compressible flow, the displacement-to-pressure operator assumes the form of an integro-differential operator. We derived the Fourier symbol of this operator, and we showed that in the highfrequency limit corresponding to short time intervals, this Fourier symbol can be associated with an added mass proportional to the length of the considered time interval. For the incompressible flow, the Fourier symbol represents a time-independent added mass in the highfrequency limit. Moreover, we showed for the incompressible flow that the viscous and convective effects are subordinate to the added-mass effect in the high-frequency limit.

The distinct proportionalities of the added-mass to the time step for compressible and incompressible flows yield essentially different behaviour of the subiteration method for fluid-structure-interaction problems. For compressible flows, for any setting of the density of the fluid and the mass of the structure, the subiteration process is stable and convergent for sufficiently small time steps. Furthermore, if the time step in the time-integration method is reduced by a certain factor, then the convergence rate of the subiteration method improves by that same factor. For incompressible flows, this is not the case, and the subiteration method can be unstable even in the limit of vanishing time-step size.

Finally, we considered the implications of the difference in the convergence behaviour of the subiteration method for staggered time-integration methods. We showed that for compressible flows, the order of accuracy of a staggered method is identical to that of the underlying monolithic method, provided that a suitable predictor is used. If two subiterations per time step are applied instead of one, then the approximation provided by the staggered method approaches the monolithic result in the limit of vanishing time-step size. Moreover, we showed that for 
compressible flows, staggered time-integration methods are stable in the limit of vanishing time-step size, in the sense that the solution remains bounded in finite-time. For incompressible flows, the order of accuracy of a stable staggered approximation with prediction is one order lower than the corresponding monolithic result. Moreover, for incompressible flows, time-integration schemes with a finite number of subiterations per time step can be unstable in the limit of vanishing time-step size, in the sense that the approximation can grow unbounded in finite time, if the subiteration process converges too slowly. Staggered methods therefore appear appropriate for fluid-structure-interaction problems with compressible flows, but for fluid-structure-interaction problems with incompressible flows their use should be dissuaded.
[1] C. Farhat, P. Geuzaine, and G. Brown, Computers and Fluids 32, 3 (2003).

[2] C. Farhat, in Encyclopedia of Computational Mechanics, edited by E. Stein, R. Borst, and T. Hughes (John Wiley \& Sons, 2004), vol. 3, chap. 13, pp. 459-480.

[3] R. Torii, M. Oshima, T. Kobayashi, K. Takagi, and T. Tezduyar, Comp. Mech. 38, 482 (2006).

[4] T. Tezduyar, S. Sathe, T. Cragin, B. Nanna, B. Conklin, J. Pausewag, and M. Schwaab, Int. J. Num. Meth. Fluids 54, 901 (2007).

[5] C. Michler, H. van Brummelen, and R. de Borst, Comput. Methods Appl. Mech. Engrg. 195, 2124 (2006).

[6] M. Heil, Comput. Methods Appl. Mech. Engrg. 193, 1 (2004).

[7] H. van Brummelen, K. van der Zee, and R. de Borst, Appl. Num. Math. (2007), DOI: 10.1016/j.apnum.2007.11.012.

[8] S. Piperno and C. Farhat, Comput. Methods Appl. Mech. Engrg. 190, 3147 (2001).

[9] C. Felippa, K. Park, and C. Farhat, Comput. Methods
Appl. Mech. Engrg. 190, 3247 (2001).

[10] P. Causin, J. Gerbeau, and F. Nobile, Comp. Meth. Appl. Mech. Engng. 194, 4506 (2005).

[11] P. LeTallec and J. Mouro, Comput. Methods Appl. Mech. Engrg. 190, 3039 (2001).

[12] C. Förster, W. Wall, and E. Ramm, Comput. Meth. Appl. Mech. Engrg. 196, 1278 (2007).

[13] T. Tezduyar, S. Sathe, R. Keedy, and K. Stein, Comput. Methods Appl. Mech. Engrg. 195, 2002 (2006).

[14] T. Tezduyar, Comput. Methods Appl. Mech. Engrg. 195, 2002 (2006).

[15] E. Zauderer, Partial Differential Equations of Applied Mathematics, Pure and Applied Mathematics (Wiley, Chichester, 1989), 2nd ed.

[16] R. Haberman, Applied Partial Differential Equations (Pearson Prentice Hall, Upper Saddle River, 1998), 3rd ed.

[17] H. van Brummelen and R. de Borst, SIAM J. Sci. Comput. 27, 599 (2005). 